$\begin{array}{ll}\text { FIGITAL } & \text { Frontiers } \\ \text { Mnest and } \\ \text { Mass ranser }\end{array}$

\title{
NON-SIMILAR SOLUTION OF A STEADY COMPRESSIBLE BOUNDARY LAYER FLOW OVER A THIN CYLINDER
}

\author{
S.V. Subhashini ${ }^{\mathrm{a}, *}$, Nancy Samuel ${ }^{\mathrm{b}}$ \\ ${ }^{a}$ Department of Mathematics, Anna University, Chennai, 600025, India \\ ${ }^{b}$ Department of Mathematics, M. S. Ramaiah Institute of Technology, Bangalore, 560054, India
}

\begin{abstract}
The aim of this paper is to present non-similar solutions of a steady laminar compressible boundary layer flow past a long thin circular cylinder including the effects of wall enthalpy and surface mass transfer. The governing equations along with the boundary conditions are first converted into dimensionless form by a non-similar transformation, and then the resulting system of coupled non-linear partial differential equations is solved by an implicit finite difference scheme in combination with the quasi-linearization technique. The increase in the value of power law variation of viscosity causes an increase in the boundary layer thicknesses of both the velocity and enthalpy profiles. Skin friction as well as heat transfer at the wall increases with suction and decreases with injection. The increase in viscous dissipation causes an increase in skin friction and decrease in heat transfer at the wall.
\end{abstract}

Keywords: Non-similar solutions, compressible flow, suction/injection, power law variation of viscosity.

\section{INTRODUCTION}

When the fluid moves at a speed comparable to the speed of sound, density changes become more significant and the flow is termed as compressible. Compressible flow deals with more than air, including steam, natural gas, nitrogen and helium, etc. Compressible flow also includes flow around bodies such as the wings of an airplane and such flows are termed as external flows. Compressible flows are also experienced in manufacturing process such as die casting and injection molding. The die casting process is a process in which liquid metal is injected into a mold to obtain a near final shape. The air is displaced by the liquid metal in a very rapid manner and therefore compressibility has to be taken into account. Also, cooling of some manufacturing systems and design of refrigeration systems utilize compressed air flow. References (Subhashini and Nath, 1999; Subhashini et al., 2003; Chamkha et al., 2005; Kumari and Nath, 2005; Bryuno and Shadrina, 2007) give information on some of the recent works on compressible boundary layer flows for various geometries.

Compressible boundary layer flow over two dimensional or axisymmetric bodies provide many practical applications. For example, two dimensional or axisymmetric bodies simulate approximately the leading edge of an aircraft or an airplane body surface and at the same time also allow a basic simplification of the complicated three dimensional compressible boundary layer equations. For axisymmetric flow, both the velocity and thermal boundary layer thicknesses are assumed to be small compared to either the body radius for an external flow or the channel radius for an internal flow. Bryuno and Shadrina (2007) have used methods of power geometry to study the axisymmetric boundary layer on a needle. Also, Roy (2000), Roy and Takhar (2003) have analyzed the effect of non-uniform multiple slot injection and wall enthalpy on the steady non-similar compressible boundary layer flow over axisymmetric bodies. Reddy (2014) has used Crank-Nicolson finite difference scheme to study the boundary layer of vertical cylinder. Similarity solutions of mixed convection boundary layer flows are also presented in a recent study by Daba et.al (2015). Experimental investigation of the boundary layer transition on an axisymmetric model is given by Sugiura et al. (2006). The mass transfer from wall into the boundary layer is of great interest for various potential applications including thermal protections, energizing the inner portion of boundary layer in adverse pressure gradient and skin friction reduction on control surfaces. In fact, mass transfer influences the development of boundary layer and can prevent or at least delay the point of separation.

The aim of the present investigation is to analyze the effect of wall enthalpy and mass transfer on steady laminar boundary layer flow of viscous compressible fluids past a long thin circular cylinder. Owing to the extreme complexity of the fully compressible boundary layer equations a mathematical model is adopted which can be justified to a certain extent on physical grounds. In short, the assumption is made that the effects of compressibility are confined to the boundary layer and the main stream remains incompressible. This could be realized in practice by releasing a stream of small Mach number past a very hot body. The nonsimilar solution of the boundary layer equations has been obtained numerically using the method of quasi-linearization in combination with an implicit finite difference scheme (Singh and Roy, 2007; Srinivasacharya et al., 2015). The results for some particular cases are compared and found to agree with the available results in literature $(\mathrm{Na}$ and Pop, 1999; Kumari and Nath, 2004).

*Corresponding author. Email: subha@annauniv.edu 


\section{ANALYSIS}

A steady axisymmetric boundary layer flow of a compressible fluid with variable properties past a static thin cylinder of infinite length and radius $b$ is considered. Let $x$ and $y$ be the curvilinear coordinates along and perpendicular to the boundary, respectively, $u$ and $v$ be the corresponding velocity components. $r=b+y$ is the normal distance from the axis of the cylinder (see Fig. 1). It is also assumed that the injected fluid possesses the same physical properties as the boundary layer fluid and has a static temperature equal to the wall temperature.

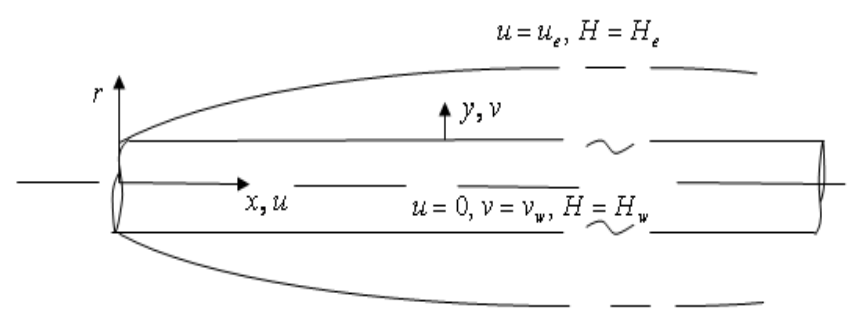

Fig. 1 Physical model and coordinate system

The Prandtl number ( $\mathrm{Pr})$ is assumed to be constant as its variation across the boundary layer is negligible for most atmospheric problems (Wortman et al., 1971). Also the external flow is homo-entropic and the surface is maintained at a constant temperature. Under the above assumptions the governing boundary layer equations are

$\frac{\partial}{\partial x}(\rho r u)+\frac{\partial}{\partial y}(\rho r v)=0$

$\rho\left(u \frac{\partial u}{\partial x}+v \frac{\partial u}{\partial y}\right)=-\frac{\partial p}{\partial x}+\frac{1}{r} \frac{\partial}{\partial y}\left(r \mu \frac{\partial u}{\partial y}\right)$

$\rho\left(u \frac{\partial h}{\partial x}+v \frac{\partial h}{\partial y}\right)=u \frac{\partial p}{\partial x}+\frac{1}{r} \frac{\partial}{\partial y}\left(\frac{\mu r}{\operatorname{Pr}} \frac{\partial h}{\partial y}\right)+\mu\left(\frac{\partial u}{\partial y}\right)^{2}$,

with boundary conditions

$u(x, 0)=0, v(x, 0)=v_{w}, h(x, 0)=h_{w}$,
$u(x, \infty)=u_{e}, h(x, \infty)=h_{e}$.

Here $h\left(h=C_{P} T\right)$ is the specific enthalpy of the fluid, $u_{e}$ and $h_{e}$ are constants, and $\partial p / \partial x=0$. It is assumed that the fluid properties vary as $\rho \propto h^{-1}$ and $\mu \propto h^{\omega}$. Equations (2) and (3) are written in terms of total enthalpy $H$ as

$$
\begin{aligned}
& \rho\left(u \frac{\partial u}{\partial x}+v \frac{\partial u}{\partial y}\right)=\frac{1}{r} \frac{\partial}{\partial y}\left(r \mu \frac{\partial u}{\partial y}\right), \\
& \rho\left(u \frac{\partial H}{\partial x}+v \frac{\partial H}{\partial y}\right)=\frac{1}{r} \frac{\partial}{\partial y}\left(\frac{\mu r}{\operatorname{Pr}} \frac{\partial H}{\partial y}\right)-\frac{1}{r} \frac{\partial}{\partial y}\left[\left(\frac{1}{\operatorname{Pr}}-1\right) \mu r u \frac{\partial u}{\partial y}\right],
\end{aligned}
$$

subject to boundary conditions

$$
\begin{aligned}
& u(x, 0)=0, v(x, 0)=v_{w}, H(x, 0)=H_{w}, \\
& u(x, \infty)=u_{e}, H(x, \infty)=H_{e} .
\end{aligned}
$$

Further, introducing new variables (Choi, 1982)

$\bar{x}=x, \bar{y}=y+\frac{y^{2}}{2 b}, \quad r=b+y, \bar{u}(\bar{x}, \bar{y})=u(x, y)$,

$\bar{v}(\bar{x}, \bar{y})=\frac{r}{b} v(x, y), \bar{H}(\bar{x}, \bar{y})=H(x, y)$, the equation of continuity is satisfied if the streamfunction $\psi$ is defined as

$\bar{u}=\frac{1}{b}\left(\frac{\rho_{e}}{\rho}\right) \frac{\partial \psi}{\partial \bar{y}}, \quad \bar{v}=-\frac{1}{b}\left(\frac{\rho_{e}}{\rho}\right) \frac{\partial \psi}{\partial \bar{x}}$.

Using (8), Eqs. (5) and (6) can be written as

$$
\begin{aligned}
\rho\left(\bar{u} \frac{\partial \bar{u}}{\partial \bar{x}}+\bar{v} \frac{\partial \bar{u}}{\partial \bar{y}}\right) & =\frac{\partial}{\partial \bar{y}}\left[\left(1+2 \frac{\bar{y}}{b}\right) \mu \frac{\partial \bar{u}}{\partial \bar{y}}\right], \\
\rho\left(\bar{u} \frac{\partial \bar{H}}{\partial \bar{x}}+\bar{v} \frac{\partial \bar{H}}{\partial \bar{y}}\right)=\frac{\partial}{\partial \bar{y}}\left[\frac{1}{\operatorname{Pr}} \mu\left(1+2 \frac{\bar{y}}{b}\right) \frac{\partial \bar{H}}{\partial \bar{y}}\right] & -\frac{\partial}{\partial \bar{y}}\left[\left(\frac{1}{\operatorname{Pr}}-1\right)\left(1+2 \frac{\bar{y}}{b}\right) \mu \bar{u} \frac{\partial \bar{u}}{\partial \bar{y}}\right],
\end{aligned}
$$

subject to boundary conditions

$\bar{u}(\bar{x}, 0)=0, \bar{v}(\bar{x}, 0)=\bar{v}_{w}, \bar{H}(\bar{x}, 0)=\bar{H}_{w}$,

$\bar{u}(\bar{x}, \infty)=\bar{u}_{e}, \quad \bar{H}(\bar{x}, \infty)=\bar{H}_{e}$.

Equations (10) and (11) are further transformed into non-dimensional form by introducing the following transformation

$\eta=\left(\frac{\bar{u}_{e}}{v_{e} \bar{x}}\right)^{1 / 2} \int_{0}^{\bar{y}} \frac{\rho}{\rho_{e}} d \bar{y}, \xi=\frac{\bar{x}}{b}, f(\xi, \eta)=\frac{1}{b}\left(v_{e} \bar{u}_{e} \xi b\right)^{-1 / 2} \psi(\bar{x}, \bar{y})$,
$G=\frac{\bar{H}}{\bar{H}_{e}}, \bar{u}=\bar{u}_{e} f^{\prime}(\xi, \eta), \bar{v}=-\frac{\rho_{e}}{\rho}\left(v_{e} \bar{u}_{e}\right)^{1 / 2}(\xi b)^{-1 / 2}\left[\frac{f}{2}+\xi \frac{\partial f}{\partial \xi}\right]$.

Thus, Eqs. (10) - (11) become

$$
\begin{aligned}
& \frac{\partial}{\partial \eta}\left[\left(1+\frac{2 Y}{b}\right) N F_{\eta}\right]+\frac{f}{2} F_{\eta}=\xi\left[F F_{\xi}-f_{\xi} F_{\eta}\right], \\
& \frac{\partial}{\partial \eta}\left[\frac{1}{\operatorname{Pr}}\left(1+\frac{2 Y}{b}\right) N G_{\eta}\right]+\frac{f}{2} G_{\eta} \\
& \quad+\operatorname{Ec} \frac{\partial}{\partial \eta}\left[\left(1-\frac{1}{\operatorname{Pr}}\right)\left(1+\frac{2 Y}{b}\right) 2 N F F_{\eta}\right]=\xi\left[F G_{\xi}-G_{\eta} f_{\xi}\right]
\end{aligned}
$$

where $\frac{\rho_{e}}{\rho}=\frac{\bar{h}}{\bar{h}_{e}}=\frac{\bar{H}-\frac{1}{2} \bar{u}^{2}}{\bar{H}_{e}-\frac{1}{2} \bar{u}_{e}{ }^{2}}=\frac{G-\mathrm{Ec} F^{2}}{1-\mathrm{Ec}}, \frac{\mu}{\mu_{e}}=\left(\frac{\bar{h}}{\bar{h}_{e}}\right)^{\omega}$,

$N=\frac{\rho \mu}{\rho_{e} \mu_{e}}=\left(\frac{G-\mathrm{Ec} F^{2}}{1-\mathrm{Ec}}\right)^{\omega-1}, \mathrm{Ec}=\frac{1}{2} \frac{\bar{u}_{e}^{2}}{\bar{H}_{e}}, \frac{\bar{y}}{b}=\frac{Y}{b}=\operatorname{Re}^{-1 / 2} \xi^{1 / 2} \int_{0}^{\eta} \frac{\rho_{e}}{\rho} d \eta$.

Here $\omega$ is the power law variation of viscosity, $\omega=0.5$ represents high temperature flows, $\omega=0.7$ is appropriate for low temperature flows and $\omega=1.0$ represents constant density-viscosity product simplifications. $N$ is the density-viscosity product, Ec is the Eckert number, Re is the Reynolds number and $\operatorname{Pr}$ is the Prandtl number which is taken as constant across the boundary layer. The boundary conditions imposed on the Eqs. (14) - (15) for $\xi \geq 0$ are

$f=f_{w}, \quad F=0, \quad G=G_{w} \quad$ at $\quad \eta=0$

$F \rightarrow 1, \quad G \rightarrow 1 \quad$ as $\quad \eta \rightarrow \infty$

where $f=\int_{0}^{\eta} F d \eta+f_{w}$

$f_{w}$ is given by $A=-\left(\rho_{e} / \rho\right)\left(v_{e} \bar{u}_{e} / \xi b\right)^{1 / 2}\left[f_{w} / 2+\xi\left(f_{\xi}\right)_{w}\right]$. The mass transfer parameter $A>0$ and $A<0$ according as there is suction or injection. 
The local skin friction coefficient and the local heat transfer coefficient in the form of Stanton number are defined as

$C_{f x}=\frac{\left[\mu \frac{\partial \bar{u}}{\partial \bar{y}}\right]_{w}}{\rho_{e} \bar{u}_{e}^{2}}=\left(\operatorname{Re}_{x}\right)^{-1 / 2}\left(\bar{F}_{\eta}\right)_{w}$

where $\left(\bar{F}_{\eta}\right)_{w}=N_{w}\left(F_{\eta}\right)_{w}$ and $\operatorname{Re}_{x}$ is the local Reynolds number.

$\mathrm{St}_{x}=\frac{\left[\frac{k}{C_{p}} \frac{\partial \bar{H}}{\partial \bar{y}}\right]_{w}}{\left(\bar{H}_{e}-\bar{H}_{w}\right) \rho_{e} \bar{u}_{e}}=\left(\operatorname{Re}_{x}\right)^{-1 / 2}\left(\bar{G}_{\eta}\right)_{w}$

where $\left(\bar{G}_{\eta}\right)_{w}=N_{w}\left(G_{\eta}\right)_{w} /\left[\operatorname{Pr}\left(1-G_{w}\right)\right]$. It is clear from Eqs. (17) - (18) that $\left(\bar{F}_{\eta}\right)_{w}$ and $\left(\bar{G}_{\eta}\right)_{w}$ are the crucial parameters which characterize the skin friction and heat transfer of the fluid flow.

\section{METHOD OF SOLUTION}

The set of dimensionless non-linear coupled partial differential equations (14), (15) under the boundary conditions (16) has been solved numerically using an implicit finite-difference scheme in combination with the quasi-linearization technique. The quasi-linearization technique is essentially a generalized Newton-Raphson method for the functional equations. The advantage of this technique is its quadratic convergence property. A detailed treatment of the quasi-linearization technique can be found elsewhere (Patil and Roy, 2010). An iterative sequence of linear equations is carefully constructed to approximate the nonlinear equations (14), (15) for achieving quadratic convergence. Applying the quasi-linearization technique, the nonlinear coupled system of partial differential equations is replaced by the following sequence of linear partial differential equations

$$
\begin{aligned}
F_{\eta \eta}^{i+1}+X_{1}^{i} F_{\eta}^{i+1}+X_{2}^{i} F^{i+1}+X_{3}^{i} F_{\xi}^{i+1}+X_{4}^{i} G_{\eta}^{i+1} & +X_{5}^{i} G^{i+1}=X_{6}^{i}, \\
G_{\eta \eta}^{i+1}+Y_{1}^{i} G_{\eta}^{i+1}+Y_{2}^{i} G^{i+1}+Y_{3}^{i} G_{\xi}^{i+1}+Y_{4}^{i} F_{\eta \eta}^{i+1} & +Y_{5}^{i} F_{\eta}^{i+1} \\
& +Y_{6}^{i} F^{i+1}=Y_{7}^{i} .
\end{aligned}
$$

The coefficient functions with iterative index $i$ are known and the functions with iterative index $(i+1)$ are to be determined. The boundary conditions are given by

$$
\begin{aligned}
& F^{i+1}=0, \quad G^{i+1}=G_{w}, \quad \text { at } \quad \eta=0, \\
& F^{i+1}=1, \quad G^{i+1}=1, \quad \text { at } \quad \eta=\eta_{\infty} \text {, }
\end{aligned}
$$

where $\eta_{\infty}$ is the edge of the boundary layer. The coefficients in equations (19), (20) are given by

$$
\begin{aligned}
& X_{1}^{i}=(\omega-1) P^{-1}\left(P_{3}-2 \mathrm{Ec} F F_{\eta}\right)+\frac{\left(2(\xi / \mathrm{Re})^{1 / 2} P^{\omega} R^{-\omega}+(f / 2)+\xi f_{\xi}\right)}{S P^{\omega-1} R^{1-\omega}}, \\
& X_{2}^{i}=-2 \mathrm{Ec}(\omega-1) P^{-1}\left(F T_{1}+F_{\eta}^{2}\right)-\frac{\xi F_{\xi}+4 \mathrm{Ec}(\xi / \operatorname{Re})^{1 / 2} P^{\omega-1} R^{-\omega}\left(\omega F F_{\eta}+\left(f-f_{w}\right) T\right)}{S P^{\omega-1} R^{1-\omega}}, \\
& X_{3}^{i}=-\frac{\xi F}{S P^{\omega-1} R^{1-\omega}}, \quad X_{4}^{i}=(\omega-1) F_{\eta} P^{-1}, X_{5}^{i}=(\omega-1) P^{-1} T_{1}+\frac{2(\xi / \operatorname{Re})^{1 / 2}\left(\omega F_{\eta}+\eta T\right)}{S R}, \\
& X_{6}^{i}=P_{2} T_{1}+F_{\eta} P_{4}-\frac{\xi F F_{\xi}}{S P^{\omega-1} R^{1-\omega}}+\frac{2(\xi / \operatorname{Re})^{1 / 2} P^{\omega-1} R^{-\omega}\left(\omega P_{2} F_{\eta}-2 \mathrm{Ec}\left(f-f_{w}\right) F T+\eta G T\right)}{S P^{\omega-1} R^{1-\omega}}, \\
& Y_{1}^{i}=(\omega-1) P^{-1}\left(P_{3}+L\right)+\frac{2(\xi / \operatorname{Re})^{1 / 2} P^{\omega} R^{-\omega}+\operatorname{Pr}(f / 2)+\xi \operatorname{Pr} f_{\xi}}{S P^{\omega-1} R^{1-\omega}}, \\
& Y_{2}^{i}=(\omega-1) P^{-1} L_{1}+\frac{2(\xi / \operatorname{Re})^{1 / 2}\left(\eta L_{2}+\omega L\right)}{S R},
\end{aligned}
$$

$$
\begin{aligned}
Y_{3}^{i}=- & \frac{\xi \operatorname{Pr} F}{S P^{\omega-1} R^{1-\omega}}, Y_{4}^{i}=E_{1} F, \\
Y_{5}^{i}=2 \operatorname{Ec}(\omega-1) F P^{-1}\left((\operatorname{Pr}-1)\left(P_{3}-2 \mathrm{Ec} F F_{\eta}\right)-G_{\eta}\right) & \\
& +2 E_{1} F_{\eta}+\frac{2 E_{1}(\xi / \operatorname{Re})^{1 / 2} P F}{R S}, \\
Y_{6}^{i}= & -2 \operatorname{Ec}(\omega-1) P^{-1}\left(F L_{1}+F_{\eta} L\right)+E_{1} T+4 \mathrm{Ec}(\xi / \operatorname{Re})^{1 / 2} P^{\omega} R^{-\omega} \\
& \quad\left(\frac{\left.(\operatorname{Pr}-1) F_{\eta}-\omega F L P^{-1}-P^{-1} L_{2}\left(f-f_{w}\right)-\xi \operatorname{Pr} G_{\xi}\right)}{S P^{\omega-1} R^{1-\omega}},\right. \\
Y_{7}^{i}= & 2(\xi / \operatorname{Re})^{1 / 2} S^{-1}\left[2 F\left(P F_{\eta}(\operatorname{Pr}-1)-\omega \operatorname{Ec} F^{2} L-\operatorname{Ec} F L_{2}\left(f-f_{w}\right)\right)\right. \\
& \left.+\eta L_{2} G+\omega L G\right]+(\omega-1) P^{-1}\left(L P_{3}+L_{1} P_{2}+2 \mathrm{Ec} F F_{\eta}\left(G_{\eta}-E_{1} F F_{\eta}\right)\right) \\
& +E_{1}\left(F_{\eta}^{2}+T F\right)-\frac{\xi \operatorname{Pr} F G_{\xi}}{S P^{\omega-1} R^{1-\omega}},
\end{aligned}
$$

where $P=\left(G-\mathrm{Ec} F^{2}\right), P_{2}=\left(G-2 \mathrm{Ec} F^{2}\right), P_{3}=\left(G_{\eta}-2 \mathrm{Ec} F F_{\eta}\right)$,

$P_{4}=\left(G_{\eta}-4 \mathrm{Ec} F F_{\eta}\right), R=(1-\mathrm{Ec}), S=1+2\left(\frac{\xi}{\operatorname{Re}}\right)^{1 / 2} \int_{0}^{\eta} \frac{P}{R} d \eta$,

$T=F_{\eta \eta}+(\omega-1) P^{-1} P_{3} F_{\eta}, T_{1}=F_{\eta \eta}+(\omega-2) P^{-1} P_{3} F_{\eta}$,

$K_{1}=G_{\eta \eta}+(\omega-2) P^{-1} P_{3} G_{\eta}, K_{2}=G_{\eta \eta}+(\omega-1) P^{-1} P_{3} G_{\eta}$,

$E_{1}=2 \mathrm{Ec}(\operatorname{Pr}-1), L=G_{\eta}+E_{1} F F_{\eta}$,

$L_{1}=K_{1}+E_{1}\left(F T_{1}+F_{\eta}^{2}\right), L_{2}=K_{2}+E_{1}\left(F T+F_{\eta}^{2}\right)$.

The resulting sequence of linear partial differential equations (19), (20) was discretized using second order central difference formula in $\eta$ direction and backward difference formula in $\xi$ - direction. In each iteration step, the equations were then reduced to a system of linear algebraic equations, with a block tri-diagonal structure which is solved using Varga's algorithm (2000). Since the method is elegantly presented in a recent study by Patil and Roy (2010) for boundary layer equations, its detailed description is not provided here to conserve the space. The convergence of numerical solutions to the exact solution is ensured by optimizing the step sizes $\Delta \eta$ and $\Delta \xi$, and the optimized step sizes are $\Delta \eta=0.01$ and $\Delta \xi=0.01$. The results presented here are independent of the step sizes at least up to the fourth decimal place. A convergence criterion based on the relative difference between the current and previous iteration values is employed. The solution is assumed to have converged and the iteration process is terminated when the difference reaches $10^{-4}$ i.e.

$\operatorname{Max}\left\{\left|\left(F_{\eta}\right)_{w}^{i+1}-\left(F_{\eta}\right)_{w}^{i}\right|,\left|\left(G_{\eta}\right)_{w}^{i+1}-\left(G_{\eta}\right)_{w}^{i}\right|\right\}<10^{-4}$.

\section{RESULTS AND DISCUSSION}

Computations have been carried out for various values of $A(-1.0 \leq A \leq$ $1.0), G_{w}\left(0.4 \leq G_{w} \leq 0.6\right), \omega(0.5 \leq \omega \leq 1.0)$ and $\mathrm{Ec}(0.2 \leq \mathrm{Ec} \leq 0.6)$. In all numerical computations $\operatorname{Pr}$ has been taken as 0.7 . In order to verify the correctness of the present numerical approach, the computed results are compared with those of $\mathrm{Na}$ and Pop (1999) for compressible flow case. The incompressible flow study reported by Kumari and Nath (2004) is also possible to compare for a specific set of parameter values with the present study. The results are found to be in excellent agreement and some of the comparisons are shown in Table 1 and Fig. 2 .

Table 1 Comparison of skin friction $\left(\operatorname{Re}_{x}^{1 / 2} C_{f x}\right)$ for various values of $\xi$ when $\mathrm{Ec}=0, \operatorname{Pr}=0.7, G_{w}=0, A=0, \omega=1.0$.

\begin{tabular}{ccc}
\hline$\xi$ & Kumari and Nath (2004) & Present Result \\
\hline 0 & 1.3281 & 1.3282 \\
1 & 1.9167 & 1.9166 \\
2 & 2.3975 & 2.3975 \\
\hline
\end{tabular}




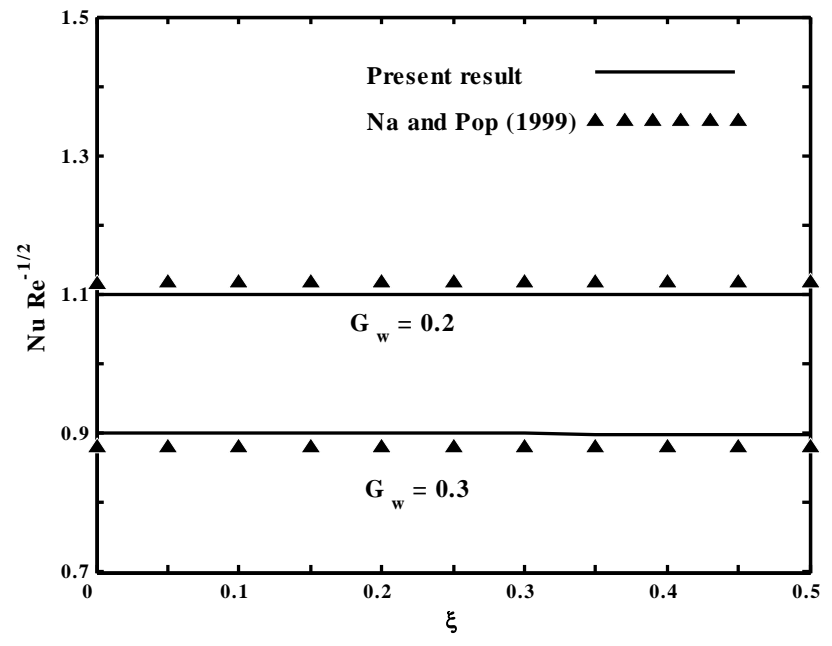

Fig. 2 Comparison of Local Nusselt number $\left(\mathrm{Nu} \mathrm{Re}^{-1 / 2}=\mathrm{St} \operatorname{Pr} \mathrm{Re}^{1 / 2}\right)$ for various values of $G_{w}$ with results of $\mathrm{Na}$ and Pop (1999) for $\operatorname{Pr}=0.7, \omega=0.5, \mathrm{Ec}=0$ and $A=0$

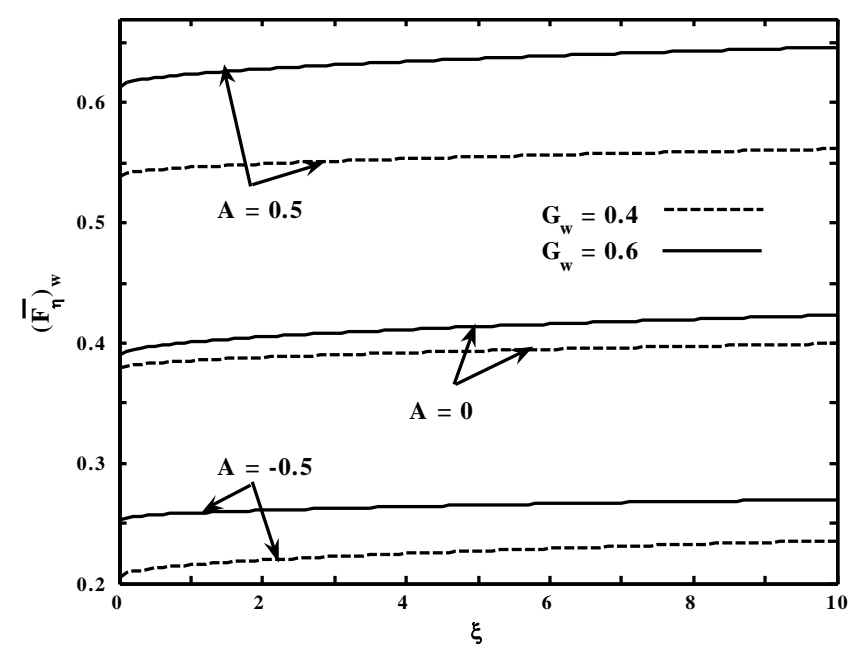

Fig. 3 Effects of $G_{w}$ and $A$ on skin friction $\left(\bar{F}_{\eta}\right)_{w}$ for $\mathrm{Ec}=0.6, \operatorname{Pr}=0.7$ and $\omega=0.5$.

The effects of wall enthalpy $\left(G_{w}\right)$ and mass transfer parameter $(A)$ on the skin friction and heat transfer rate at the wall are presented in Figs. 3 and 4. The results presented in Fig. 3 indicate that the skin friction increases with suction $(A>0)$ and decreases with injection $(A<0)$. For example, approximately $40 \%$ increase in skin friction is noticed as $A$ changes from 0 to 0.5 whereas, $32 \%$ decrease is noticed as $A$ changes from 0 to -0.5 for $G_{w}=0.4$ at $\xi=10$. The physical reasoning for the above behaviour is that blowing gives rise to a thicker momentum boundary layer thereby decreasing velocity gradient at the surface. Similarly, heat transfer at the wall increases with increase of suction and decreases with the increase of injection. It is observed from Figs. 3 and 4 that as the wall enthalpy increases, the heat transfer rate at the wall decreases while skin friction increases. This is because the fluid (air) viscosity is increased due to the increase in the wall enthalpy which results in increase of skin friction. On the other hand, as the wall enthalpy increases, the density decreases. Decrease in density results in increase of the thermal boundary layer thickness which causes reduction in heat transfer rate at the wall. From Fig. 4 it is noted that for $A=0.7$, there is approximately $30 \%$ decrease in heat transfer rate at the wall as the wall enthalpy increases from 0.4 to 0.6 at $\xi=10.0$. Similarly,
Fig. 3 indicates that skin friction increases approximately $13 \%$ for $A=-0.5$ when the wall enthalpy changes from 0.4 to 0.6 at $\xi=10.0$. Further for $A=0.5$, increase in $\left(\bar{F}_{\eta}\right)_{w}$ is $15 \%$ at $\xi=10.0$.

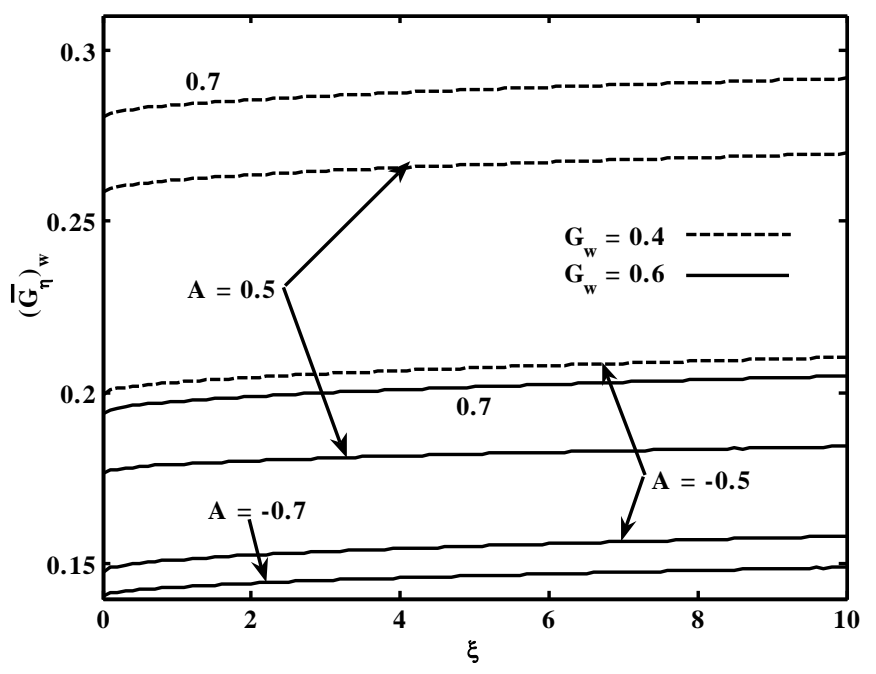

Fig. 4 Effects of $G_{w}$ and $A$ on heat transfer at the wall $\left(\bar{G}_{\eta}\right)_{w}$ for $\mathrm{Ec}=0.6, \operatorname{Pr}=0.7$ and $\omega=0.5$.

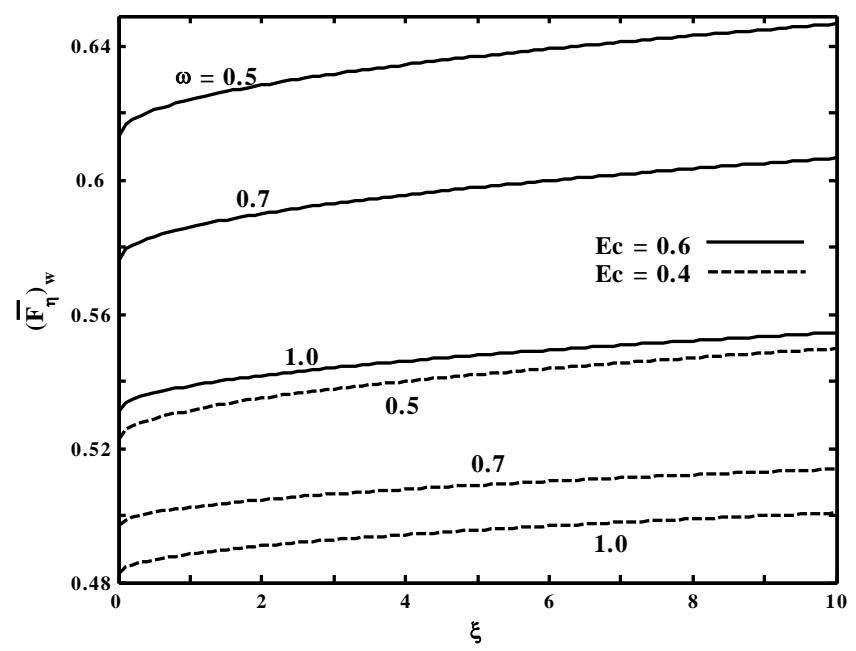

Fig. 5 Effects of Ec and $\omega$ on skin friction $\left(\bar{F}_{\eta}\right)_{w}$ for $G_{w}=0.6, \operatorname{Pr}=$ 0.7 and $A=0.5$.

Figures 5 and 6 present the effect of viscous dissipation parameter and power law variation of viscosity on skin friction and heat transfer rate at the wall, respectively. The increase in viscous dissipation (Ec) causes increase in the skin friction. It is interesting to observe in Fig. 5 that as the dissipation parameter increases from 0.4 to 0.6 there is approximately $17 \%$ increase in skin friction for $\omega=0.5$ at $\xi=10.0$. Also, it is noticed in Fig. 6 that the increase in viscous dissipation causes a decrease in heat transfer rate at the wall. This is because the kinetic energy of the flow will be partially dissipated by the influence of friction and will be transformed into internal energy of the fluid. Thus, the viscous dissipation increases the frictional heating and consequently the surface gets heated. This results into the enthalpy difference between the wall and the fluid near the wall. Hence, the enthalpy gradient becomes less and heat transfer is reduced at the wall (Fig. 6). It is noted from Figs. 5 and 6 that increase in power law 
variation of viscosity $(\omega)$ decreases both skin friction and heat transfer rate at the wall. In particular, for $\xi=5.0$ and $\mathrm{Ec}=0.6$, there is approximately $15 \%$ decrease in the skin friction as $\omega$ increases from 0.5 to 1.0 (see Fig.5). Similarly, there is about $20 \%$ decrease in heat transfer rate at the wall as $\omega$ increases from 0.5 to 1.0 at $\xi=5.0$ when $\mathrm{Ec}=0.6$ (see Fig.6).

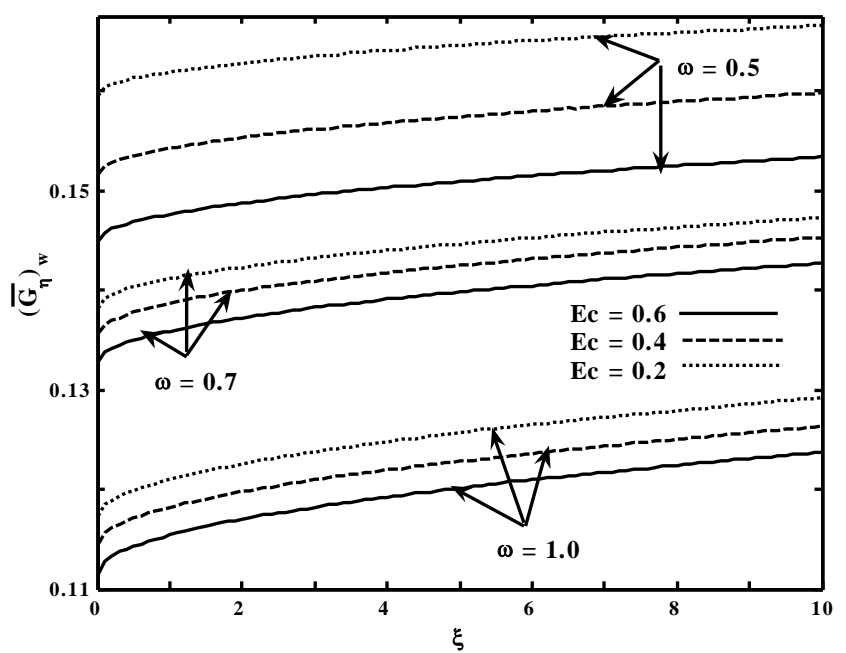

Fig. 6 Effects of Ec and $\omega$ on heat transfer at the wall $\left(\bar{G}_{\eta}\right)_{w}$ for $G_{w}=0.6, \operatorname{Pr}=0.7$ and $A=0.5$.

It may be remarked from Figs. 5 and 6 that the effect of power law variation of viscosity $(\omega)$ is slightly more pronounced on heat transfer rate at the wall as compared on skin friction. Also, it is seen from Figs. 5 and 6 that skin friction and heat transfer at the wall increases with increase in $\xi$. It is noticed in Fig. 6 that the increase in heat transfer at the wall with the increase of $\xi$ is significant for higher values of $\omega$. For example, for $\omega=1.0$, approximately $11 \%$ increase in heat transfer at the wall is noted as $\xi$ increases from 0 to 10.0 whereas only $4 \%$ increase in $\left(\bar{G}_{\eta}\right)_{w}$ is observed for $\omega=0.5$ when $\mathrm{Ec}=0.6$.

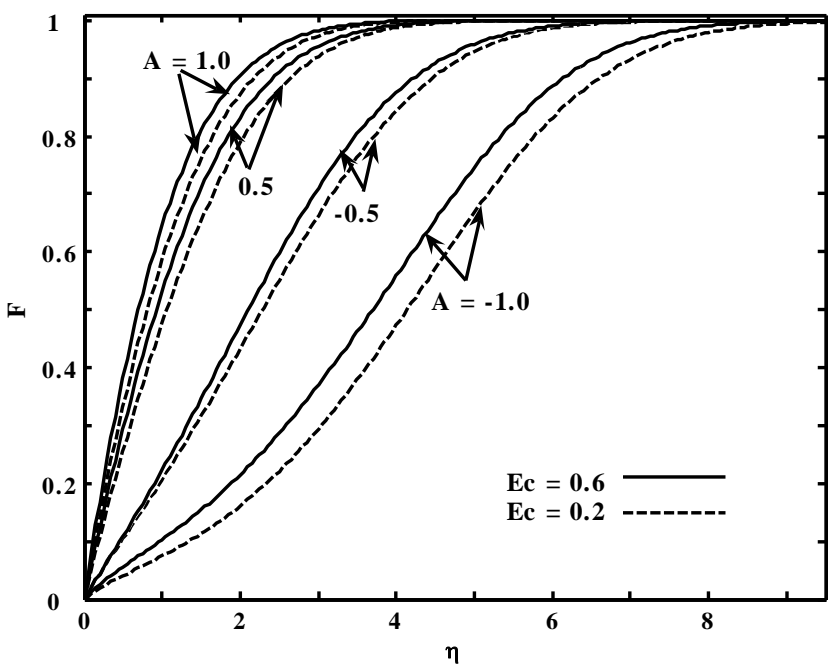

Fig. 7 Effects of Ec and $A$ on velocity profile $(F)$ for $G_{w}=0.6, \operatorname{Pr}=$ 0.7 and $\omega=0.5$ at $\xi=5.0$.

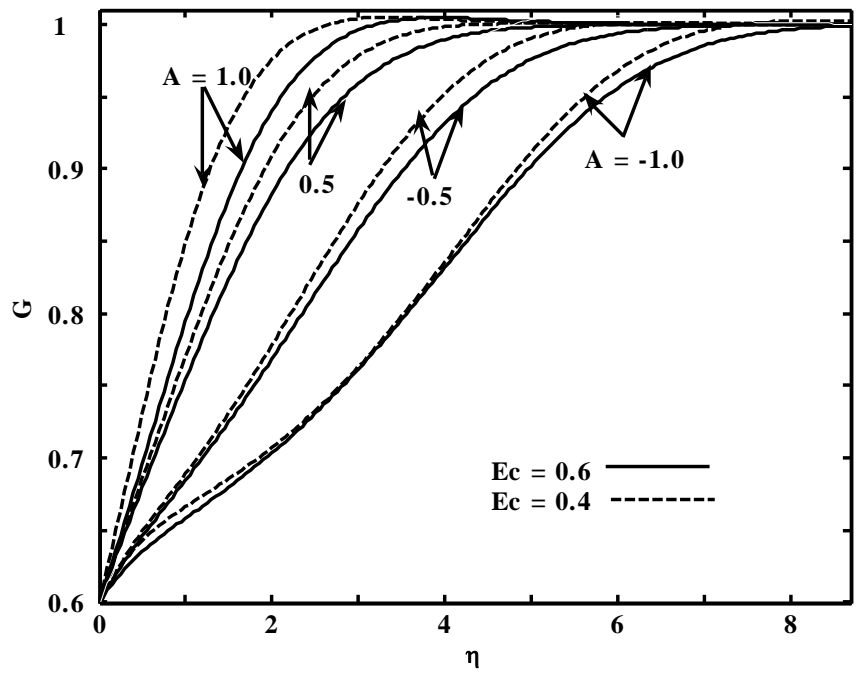

Fig. 8 Effects of Ec and $A$ on enthalpy profile $(G)$ for $G_{w}=0.6$, $\operatorname{Pr}=0.7$ and $\omega=0.5$ at $\xi=5.0$.

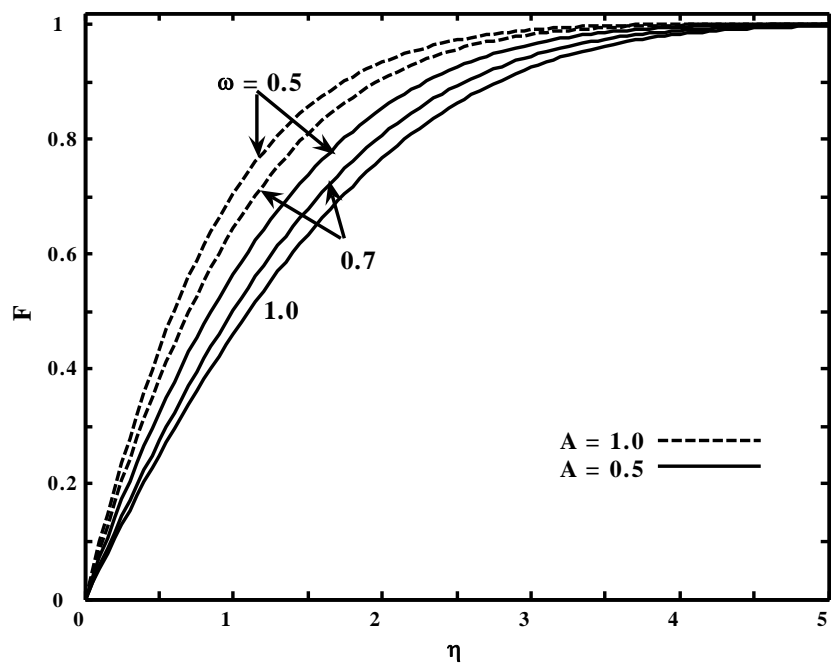

Fig. 9 Effects of $\omega$ and $A$ on velocity profile $(F)$ for $G_{w}=0.6, \operatorname{Pr}=0.7$ and $\mathrm{Ec}=0.6$ at $\xi=5.0$.

Effects of dissipation parameter and mass transfer parameter on the velocity and enthalpy profiles for $G_{w}=0.6$ and $\omega=0.5$ are presented in Figs. 7 and 8. It is noticed from Fig. 7 that the increase in the value of the dissipation parameter causes increase in the steepness of the velocity profile $(F)$, that is, the boundary layer thickness of the velocity profile decreases with the increase in the value of dissipation parameter. This is because increase in dissipation causes an increase in kinetic energy of the particles within the boundary layer thereby decreasing its thickness. Also, from Fig. 7 it is observed that the boundary layer thickness increases with injection and decreases with suction. This is due to the reason that the fluid is carried away from the surface in the case of injection $(A<0)$ causing a reduction in the velocity gradient as it tries to maintain the same velocity over a small region near the surface, and such effect is reversed in the case of suction $(A>0)$. Similarly, the enthalpy boundary layer is thick for injection and gets thinner for suction (see Fig. 8). The reason is that the fluid is brought closer to the surface by suction and hence the enthalpy boundary layer thickness is reduced. Also, it is observed from Fig. 8 that the increase in the value of dissipation parameter causes an increase in the boundary layer thickness of the enthalpy profiles. The effect of power law variation of viscosity on velocity and enthalpy profiles is 
presented in Figs. 9 and 10, respectively. It is noted that the boundary layer thickness of both velocity and enthalpy profiles increases with the increase in the value of $\omega$ (see Figs. 9 and 10). For example, it is noted from Fig. 9 that $\eta_{\infty} \approx 3.9$ and $\eta_{\infty} \approx 4.6$ for $\omega=0.5$ and $\omega=1.0$, respectively, when $A=0.5$. Similarly, Fig. 10 displays that for $A=0.5$, the enthalpy boundary layer thicknesses are approximately 3.2 and 3.4 corresponding to $\omega=0.5$ and 0.7 , respectively.

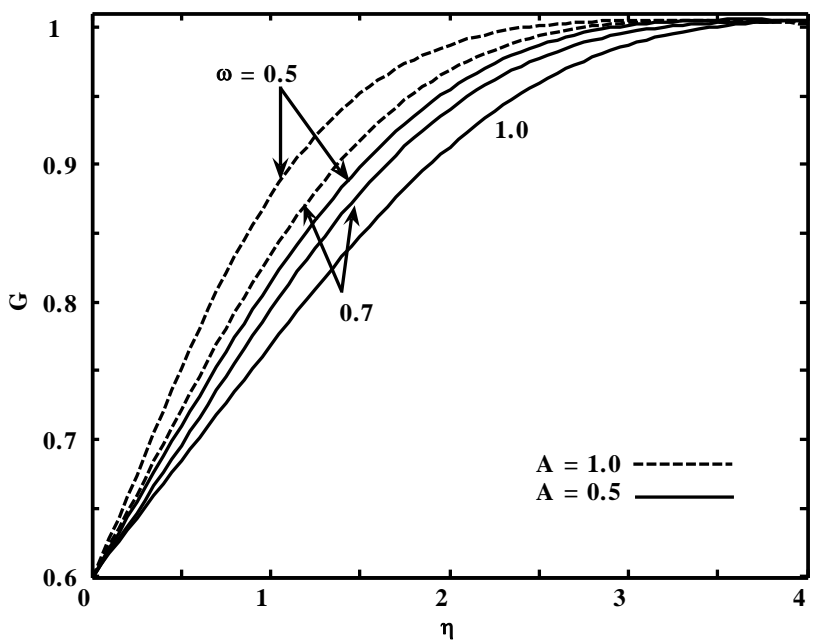

Fig. 10 Effects of $\omega$ and $A$ on enthalpy profile $(G)$ for $G_{w}=0.6$, $\operatorname{Pr}=0.7$ and $\mathrm{Ec}=0.6$ at $\xi=5.0$.

\section{CONCLUSIONS}

A steady axisymmetric boundary layer flow of a compressible fluid with variable properties past a static thin cylinder of infinite length and radius $b$ has been studied numerically. Conclusions of the study are as follows

- Results indicate that increase in wall enthalpy $G_{w}$ from 0.4 to 0.6 causes approximately $32 \%$ decrease in heat transfer at the wall $\left(\bar{G}_{\eta}\right)_{w}$ and approximately $17 \%$ increase in skin friction $\left(\bar{F}_{\eta}\right)_{w}$.

- It is noticed that skin friction increases by $40 \%$ for suction $A=0.5$ and reduces by $31 \%$ for injection $A=-0.5$. Similarly, heat transfer at the wall increases with suction and decreases with injection.

- Increase in dissipation parameter from $\mathrm{Ec}=0.4$ to $\mathrm{Ec}=0.6$ causes approximately $17 \%$ increase in skin friction and $5 \%$ decrease in heat transfer at the wall. Also, skin friction and heat transfer increase with the increase in the value of $\xi$.

- Power law variation of viscosity reduces both skin friction and heat transfer at the wall. Approximately $15 \%$ decrease in skin friction and $20 \%$ decrease in heat transfer is noticed as $\omega$ increases from 0.5 to 1.0 .

\section{NOMENCLATURE}

$r$

b

$p$

$h$

$u, v$

$x, y$

$\operatorname{Pr}$

$H$

G

$F$

$N$ normal distance from the axis of the cylinder

radius of the cylinder

pressure

specific enthalpy

velocity components in $x$ - and $y$-direction

axial and vertical coordinates

Prandtl number

total enthalpy

dimensionless enthalpy

dimensionless velocity

ratio of buoyancy parameters

$\begin{array}{ll}\text { Ec } & \text { Eckert number } \\ C_{f x} & \text { Local skin friction coefficient } \\ \mathrm{St}_{x} & \text { local Stanton number } \\ k & \text { thermal conductivity of the fluid } \\ C_{p} & \text { specific heat at constant pressure } \\ A & \text { dimensionless surface mass transfer parameter } \\ \operatorname{Re}_{x} & \text { local Reynolds number }\end{array}$

Greek Symbols

$\rho \quad$ density

$\mu \quad$ dynamic viscosity

$v \quad$ kinematic viscosity

$\eta \quad$ similarity variable

$\xi \quad$ transformed axial coordinate

$\omega \quad$ powerlaw variation in viscosity

$\psi \quad$ dimensionless stream function

Subscripts

$w \quad$ condition at the wall

$e \quad$ ambient environment

$\eta, \xi \quad$ partial derivative with respect to these variables

\section{REFERENCES}

Bryuno, A.D., and Shadrina, T.V., 2007, “An Axisymmetric Boundary Layer on a Needle," Trans. Moscow Math. Soc., 68, 201-259. http://dx.doi.org/10.1090/S0077-1554-07-00165-3

Chamkha, A.J., Takhar, H.S., and Nath, G., 2005, "Unsteady Compressible Boundary Layer Flow Over a Circular Cone Near a Plane of Symmetry," Heat and Mass Transfer, 41(7), 632-641. http://dx.doi.org/10.1007/S00231-004-0575-8

Choi, I.G., 1982, "The Effect of Variable Properties of Air on the Boundary Layer for Moving Continuous Cylinder," Int. J. Heat Mass Transfer, 25(5), 597-602. http://dx.doi.org/10.1016/0017-9310(82)90164-8

Daba, M., Devaraj, P., and Subhashini, S.V., 2015, "Mixed Convection Boundary Layer Flow Over a Vertically Stretching Sheet with Convective Boundary Condition and Effect of Slip," Frontiers in Heat and Mass Transfer, 6(3). http://dx.doi.org/10.5098/hmt.6.3

Kumari, M., and Nath, G., 2004, "Mixed Convection Boundary Layer Flow Over a Thin Vertical Cylinder with Localized Injection/Suction and Cooling/Heating," Int. J. Heat and Mass Transfer, 47(5), 969-976. http://dx.doi.org/10.1016/i.iiheatmasstransfer.2003.08.014

Kumari, M., and Nath, G., 2005, "Transient Laminar Compressible Boundary Layers Over a Permeable Circular Cone Near Plane of Symmetry," Int. J. Heat and Mass Transfer, 48(13), 2771-2778. http://dx.doi.org/10.1016/j.ijheatmasstransfer.2005.01.031

Na, T.Y., and Pop, I., 1999, "Axisymmetric Compressible Boundary Layer on a Long Thin Moving Cylinder," Acta Technica, 138(3-4), $255-260$.

http://dx.doi.org/10.1007/BF01291848

Patil, P.M., and Roy, S., 2010, "Unsteady Mixed Convection Flow from a Moving Vertical Plate in a Parallel Free Stream: Influence of Heat Generation or Absorption," Int. J. Heat Mass Transfer, 53(21), 4749-4756. http://dx.doi.org/ 10.1016/j.ijheatmasstransfer.2010.06.017.

Reddy, M. G., 2014, "Radiation Effects on MHD Natural Convection Flow Along a Vertical Cylinder Embedded in a Porous Medium with Variable Surface Temperature and Concentration," Frontiers in Heat and Mass Transfer, 5 - 013004.

http://dx.doi.org/10.5098/hmt.5.4 
Roy, S., 2000, "Nonuniform Multiple Slot Injection (Suction) or Wall Enthalpy into a Steady Compressible Laminar Boundary Layer," Acta Mechanica, 143(1), 113-128.

http://dx.doi.org/10.1007/BF01250021

Roy, S., and Takhar, H.S., 2003, "Compressible Boundary Layer Flow with Non-Uniform Slot Injection (or Suction) over i) a Cylinder and ii) a Sphere," Heat and Mass Transfer, 39(2), 139-146. http://dx.doi.org/10.1007/s00231-002-0306-y

Singh, P.J., and Roy, S., 2007, "Unsteady Mixed Convection Flow over a Vertical Cone due to Impulsive Motion," Int. J. Heat Mass Transfer, 50(6), 949-959.

http://dx.doi.org/10.1016/j.ijheatmasstransfer.2006.08.011

Srinivasacharya, D., Chamkha, A.J., Surendera, O., and Rashad, A. M., 2015, "Natural Convection on a Porous Vertical Plate in a Doubly Stratified Non-Darcy Porous Medium," Frontiers in Heat and Mass Transfer, 6(19).

http://dx.doi.org/10.5098/hmt.6.19
Subhashini, S.V., and Nath, G., 1999, "Unsteady Compressible Flow in the Stagnation Region of Two Dimensional and Axisymmetric Bodies," Acta Mechanica, 134(3), 135-145. http://dx.doi.org/10.1007/BF01312652

Subhashini, S.V., Takhar, H.S., and Nath, G., 2003, "Non Uniform Mmultiple Slot Injection (Suction) or Wall Enthalpy into a Compressible Flow Over a Yawed Circular Cylinder," Int. J. Thermal Sciences, 42(8), 749-757.

http://dx.doi.org/10.1016/S1290-0729(03)00046-2

Sugiura, H., Tokugawa, N., and Ueda, Y., 2006, "Boundary-Layer Transition on an Axisymmetric Body at Incidence at Mach 1.2," A.I.A.A. Journal, 44(5), 973-980. http://dx.doi.org/10.2514/1.8719

Varga, R.S., 2000, Matrix Iterative Analysis, Prentice Hall, New Jersey.

Wortman, A., Ziegler, H., and Soo-Hoo, G., 1971, "Convective Heat Transfer at General Three-Dimensional Stagnation Points," Int. J. Heat Mass Trans., 14(1), 149-152.

http://dx.doi.org/10.1016/0017-9310(71)90145-1 